\title{
DIDACTIC - Aplicación de la Gamificación y la Inteligencia Artificial en la Educación Virtual
}

Torres-Barreto, Martha Liliana ${ }^{\left.1{ }^{*}\right)}$; Acosta Medina, Julieth Katherin'; Álvarez-Melgarejo, Mileidy'

${ }^{1}$ Universidad Industrial de Santander, Facultad de Ingenierías Fisicomecánicas, Colombia, Bucaramanga

\section{RESUMEN}

La educación virtual ha tenido un auge en los últimos años, debido a los múltiples beneficios que ofrece. Sin embargo, esta modalidad educativa se enfrenta a grandes retos como la baja motivación, la falta de dinamismo en los cursos y las altas tasas de abandono. Es por esto que surge DIDACTIC, un proyecto que se compone de una herramienta gamificada y una plataforma de contactabilidad desarrollada con inteligencia artificial, las cuales buscan aumentar la motivación y optimizar la conexión docente-estudiante, con el fin de mejorar los procesos de enseñanza y aprendizaje y contribuir a la disminución del abandono estudiantil. En el presente artículo se describe este proyecto, sus bases teóricas, metodología y resultados obtenidos hasta ahora. Los hallazgos hasta el momento indican, que debido a los múltiples beneficios que ofrecen la gamificación y la inteligencia artificial es importante seguir implementándolas en entornos virtuales de aprendizaje

Palabras clave: educación virtual; gamificación; aprendizaje automático; inteligencia artificial.

\section{DIDACTIC - Application of Gamification and Artificial Intelligence in Virtual Education}

\section{ABSTRACT}

Virtual education has boomed in recent years, due to the multiple benefits it offers. However, this educational modality faces great challenges such as low motivation, lack of dynamism in the courses and high dropout rates. That is why DIDACTIC arises, a project that consists of a gamified tool and a contactability platform developed with artificial intelligence, which seeks to increase motivation and the teacher-student connection, in order to improve teaching and learning processes and contribute to the decrease in student dropout. In this research, describe this project, its theoretical bases, methodology and specific results so far. The findings so far indicated, due to the multiple benefits offered by gamification and artificial intelligence, it is important to continue implementing the facilities in virtual learning environments.

Keywords: virtual education; gamification; machine learning; artificial intelligence.. 


\section{INTRODUCCIÓN}

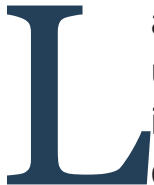

a educación virtual empezó a aparecer como una opción educativa a mediados de 2015, incluso, gracias a los avances tecnológicos desde la fecha hasta ahora, este modelo ha crecido en Colombia en un 98\% (Ministerio de Educación Nacional de Colombia, 2019). Esta modalidad educativa tiene grandes ventajas como la flexibilidad de horarios y la no restricción geográfica; sin embargo, se enfrenta a grandes retos como la necesidad de mecanismos efectivos de conexión entre docentes - estudiantes y la falta de dinamismo en los cursos, pues generalmente los entornos virtuales de aprendizaje son considerados escenarios rígidos dedicados exclusivamente a impartir conceptos (Melo-Solarte \& Díaz, 2018).

En este sentido, surge DIDACTIC, un proyecto que se basa en el desarrollo conjunto una herramienta gamificada Ilamada "Didactic City" y una plataforma de contactabilidad. La herramienta busca aplicar mecanismos asociados a los juegos, gamificando las interacciones para generar alta participación y motivación, mejorando los procesos de enseñanza y aprendizaje de competencias transversales que promuevan el desarrollo sostenible. Por su parte, la plataforma, desarrollada con inteligencia artificial, extraerá información de los sistemas de gestión de aprendizaje (LMS) que usen los programas virtuales, con el fin de establecer diferentes patrones y mensajes de comunicación hacia el estudiante, para que estos reciban mayor acompañamiento del docente, facilitando el seguimiento individual, grupal e institucional de los alumnos.

En el presente artículo se describen las bases teóricas de este proyecto, la metodología empleada y los resultados obtenidos hasta el momento.

\section{MARCO TEÓRICO}

Este proyecto se fundamenta en dos pilares: gamificación e inteligencia artificial.

\section{Gamificación}

La gamificación hace referencia al uso de elementos propios del juego en contextos que no son juegos (Deterding, Dixon, Khaled, \& Nacke, 2011). En los últimos años, la adopción de esta estrategia se ha real- izado en diversos campos como los negocios, el marketing, la gestión corporativa, la salud y la educación (Dicheva, Dichev, Agre, \& Angelova, 2015).

El impacto de la gamificación se debe, en parte, a los elementos que la componen, pues cada elemento o la combinación de estos, genera diferentes efectos psicológicos en los usuarios (Rodrigues, Oliveira, \& Rodrigues, 2019). Existen diferentes modelos conceptuales de estos elementos: MDC-Mecánicas, dinámicas y componentes; MDE-Mecánica, Dinámica y Estética; DFC- Deseo, Fantasía, Curiosidad; CEGECore elements of the gaming experience; entre otros (Acosta-Medina, Torres-Barreto, Paba-Medina, \& Alvarez-Melgarejo, 2020). Entre los elementos usados con mayor frecuencia en herramientas gamificadas se encuentran los puntos, las insignias y las tablas de clasificación (Acosta-Medina, Torres-Barreto, \& Alvarez-Melgarejo, 2020).

En las aulas presenciales y virtuales esta estrategia está ganando impulso como una innovación educativa, ya que puede motivar a los estudiantes a involucrarse en su proceso de aprendizaje, además de aumentar el engagement y contribuir a la disminución del abandono estudiantil (Acosta-Medina, Torres-Barreto, Álvarez-Melgarejo, \& Paba-Medina, 2020; Lobo-Rueba, Paba-Medina, \& Torres-Barreto, 2020).

\section{Inteligencia artificial (IA)}

La inteligencia artificial (IA) es una ciencia que estudia al cerebro humano y a la inteligencia con el objetivo de modelar matemáticamente diferentes procesos que faciliten y automaticen los problemas de diferentes áreas del conocimiento, simulando la forma y las habilidades de los seres humanos. Tiene un campo de aplicación amplio y variado, por lo que está presente en muchas áreas como: la robótica, las aplicaciones para realizar traducciones, el machine learning, entre otros (D'Addario, 2019; Galipienso, Quevedo, Pardo, Ruiz, \& Lozano, 2015). Adicionalmente, la IA es un elemento fundamental para integrar realismo a los videojuegos y a las herramientas gamificadas, pues esta le permite al jugador enfrentar un reto factible de manera que suponga un estímulo emocional, consiguiendo generar engagement al juego (Jurado, Asbusac, \& Sanchez, 2018). 
También conocido como aprendizaje automático, es una disciplina de las ciencias de la computación y una rama de la inteligencia artificial. En este, es necesario plantear un modelo matemático con algunos parámetros y gracias a la ejecución de un programa por computadora, usando datos históricos o de entrenamiento, se puede hacer predicciones para el futuro o descripciones para obtener nuevo conocimiento (Alpaydin, 2020).

Dentro de las diversas aplicaciones del machine learning, se encuentran modelos para predecir el abandono por parte de los estudiantes en cursos virtuales, siendo los modelos más usados para este propósito los árboles de decisión, la regresión logística, el análisis discriminante y las redes neuronales (Dalipi, Imran, \& Kastrati, 2018; Viloria, Senior Naveda, Hernández Palma, Niebles Núẽz, \& Niebles Núẽz, 2020). Estas predicciones son cada vez más relevantes, pues en los últimos años las tasas de abandono en programas de formación virtual son altas y oscilan en el $40 \%$, siendo del $60 \%$ para los programas académicos virtuales de Colombia (Ministerio de Educación Nacional de Colombia, 2017).

\section{METODOLOGÍA}

Tal como se dijo anteriormente, este proyecto tiene dos componentes principales: la herramienta gamificada y la plataforma de contactabilidad. A continuación, se describe la metodología usada para el diseño y desarrollo de cada uno.

\section{Herramienta gamificada "Didactic City"}

Para el diseño de "Didactic City" se sigue el enfoque metodológico propuesto por Kumar \& Herger, (2013) quienes plantean cinco pasos para el diseño iterativo de herramientas gamificadas basadas en el usuario: (1) Conocer al usuario, (2) Identificar los objetivos de aprendizaje, (3) Comprender la motivación, (4) Definir los elementos de la gamificación y (5) Administrar y monitorear.

Igualmente, para el desarrollo de esta herramienta se siguió la metodología ágil SCRUM, la cual busca desarrollar productos incrementando la flexibilidad y rapidez, a partir de un equipo de trabajo inter- disciplinario y la creación de ciclos breves llamados sprints (Takeuchi \& Nonaka, 1986). Estos ciclos se componen de cinco fases: concepto, especulación, exploración, revisión y cierre.

\section{Plataforma de contactabilidad}

Para la plataforma de contactabilidad primero se hace necesario establecer modelos machine learning con el fin de detectar patrones y comportamientos de los estudiantes. Para esto se realiza un análisis de viabilidad de diferentes modelos teniendo en cuenta diversos factores tales como: usabilidad, requerimiento de datos, adaptabilidad, precisión y lenguaje de programación.

Posterior a esto, se sigue el proceso de ETL - Extracción, Transformación y Carga de los datos desde los LMS (Canvas o Moodle) a un data warehouse, al cual se aplican los modelos machine learning desarrollados en el paso anterior. De acuerdo a los resultados de dichos modelos y con base a eventos previamente establecidos, se inicia un proceso de contacto por diferentes medios con los estudiantes que lo requieran, para que estos tengan un mayor acompañamiento por parte de los tutores o docentes. Esta plataforma se implementa utilizando el lenguaje de programación Phyton y algunas de sus librerías tales como: sklearn, pandas, numpy y scipy.

\section{RESULTADOS}

\section{Herramienta gamificada "Didactic City"}

El diseño de "Didactic City" está centrado en el usuario, por esto, primero se determinaron los perfiles de usuarios con el objetivo de conocer sus características y necesidades. Para ello, se realizó una búsqueda en la literatura y se aplicaron una serie de entrevistas abiertas, obteniéndose así cuatro perfiles de estudiantes y tres de docentes de educación virtual, los cuales se encuentran resumidos en la Tabla 1. 
Tabla 1. Perfiles de usuarios

\begin{tabular}{|c|c|}
\hline Usuario & Perfiles \\
\hline $\begin{array}{c}\text { Estudiantes de educación } \\
\text { virtual }\end{array}$ & Estudiante fantasma \\
& Estudiante trabajador \\
& Estudiante recién graduado \\
Estudiante con desafíos personales \\
\hline $\begin{array}{c}\text { Docentes de educación } \\
\text { virtual }\end{array}$ & Docente empático \\
& Docente normativo \\
& Docente activo \\
\hline
\end{tabular}

Luego, se determinaron los objetivos de aprendizaje, en este caso relacionados con las competencias ciudadanas. Teniendo en cuenta que según el Ministerio de Educación Nacional de Colombia, (2006) existen siete tipos de competencias ciudadanas, se realizó una priorización de estas, utilizando ponderación de factores y método Delphi, obteniéndose que la herramienta gamificada debía centrarse en desarrollar habilidades comunicativas escritas y competencias cognitivas de conocimiento cívico, pues estas resultan ser las más relevantes para el contexto particular de este estudio (Ver Tabla 2).

Tabla 2. Priorización de competencias ciudadanas

\begin{tabular}{|c|c|c|}
\hline Cuartil & Competencias & Puntaje \\
\hline \multirow{2}{*}{1} & Comunicativas escritas & 4,36 \\
& Cognitivas - conocimientos & 4,21 \\
\hline 2 & Comunicativas - orales & 3,65 \\
\hline \multirow{2}{*}{3} & Cognitivas - pensamiento sistémico & 3,12 \\
& Cognitivas - multiperspectivismo & 3,12 \\
& Cognitivas - argumentación & 2,92 \\
\hline \multirow{2}{*}{4} & Emocionales - manejo emociones & 2,69 \\
& Emocionales - empatía & 1,94 \\
\hline
\end{tabular}

Posteriormente, con el fin de comprender las motivaciones intrínsecas y extrínsecas de los principales usuarios, en este caso, estudiantes recién graduados, se realizaron mapas de empatía y journey maps (Ver Figura 1). Los hallazgos de estos indican que las principales motivaciones son el monitoreo y comunicación constante con el docente.

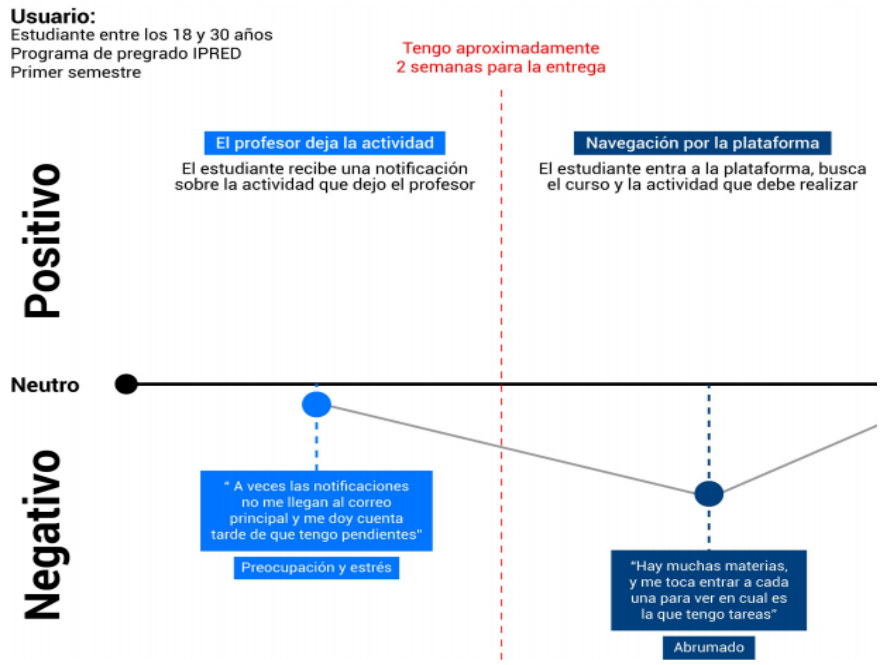

Figura 1. Journey map - Actividad: tarea

A partir de lo anterior, se definieron los elementos a incluir en la herramienta gamificada, los cuales basados en el modelo propuesto por Werbach \& Hunter, (2012) se encuentran explicados en la Tabla 3. "Didactic City" consta principalmente de una mecánica de construcción, en la que se puede ir edificando una ciudad mientras se resuelven misiones de competencias ciudadanas (preguntas saber pro, dilemas morales y situaciones problemas), por cada misión cumplida se otorgan monedas customizables dentro del juego y puntos (gamificación activa), los cuales se suman con puntos obtenidos por realizar actividades propiamente académicas dentro de los LMS como tareas o evaluaciones (gamificación pasiva). 
Tabla 3. Elementos de la gamificación de "Didactic City"

\begin{tabular}{|c|c|c|}
\hline Elemento & & Aplicación en Didactic City \\
\hline \multirow{8}{*}{ Componentes } & Avatar & $\begin{array}{c}\text { Emblemas de los } 8 \text { tipos de inteli- } \\
\text { gencia }\end{array}$ \\
\hline & Puntos & $\begin{array}{c}\text { Sistema de puntos } \\
\text { Gamificación activa: LMS } \\
\text { Gamificación pasiva: juego }\end{array}$ \\
\hline & Monedas & Monedas \\
\hline & Niveles & 8 niveles \\
\hline & Misiones & $\begin{array}{l}\text { Preguntas saber pro } \\
\text { Dilemas morales } \\
\text { Situaciones problemas }\end{array}$ \\
\hline & $\begin{array}{l}\text { Tablas de clasifi- } \\
\text { cación }\end{array}$ & $\begin{array}{c}\text { Tabla de clasificación centrada en } \\
\text { usuario }\end{array}$ \\
\hline & Barra de progreso & Barra de progreso en cada nivel \\
\hline & Límite de tiempo & $\begin{array}{l}\text { Duración cada nivel: un semestre } \\
\text { académico }\end{array}$ \\
\hline Mecánica & Construcción & Construir una ciudad \\
\hline \multirow{3}{*}{ Dinámica } & Emociones & Motivación \\
\hline & Progresión & $\begin{array}{l}\text { Niveles, monedas y barra de pro- } \\
\text { greso }\end{array}$ \\
\hline & Retroalimentación & $\begin{array}{c}\text { Feedback inmediato en las respues- } \\
\text { tas de las preguntas y los dilemas } \\
\text { morales }\end{array}$ \\
\hline
\end{tabular}

El modelo conceptual anterior se valida mediante grupos focales realizados con estudiantes de educación virtual, de los que se obtienen la necesidad de presentar situaciones complejas de la vida real en los dilemas morales y situaciones problemas, además de incorporar aspectos gráficos que les permitan a los usuarios identificarse con los avatares y las edificaciones presentadas en el juego.

Por otra parte, para la construcción de "Didactic City" se usó el lenguaje de programación C\# en el motor de Unity 3D, la interfaz gamificada se construyó bajo el enfoque web desktop, para que el juego pueda ser utilizado en ambientes web y desktop buscando favorecer el bajo tráfico y consumo de datos. El desarrollo se dividió principalmente en tres sprint: primer sprint (demo del juego), segundo sprint (juego intermedio) y tercer sprint (juego final). Para los dos primeros sprint solo se consideraron aspectos básicos de jugabilidad correspondientes al primer nivel.

Una vez terminado el demo del juego (Ver Figura $2)$, se aplicaron pruebas de usabilidad en dos momentos y con dos poblaciones diferentes (estudiantes presenciales y estudiantes virtuales). Cada prueba constaba de 30 minutos de juego en los que el usuario debía cumplir una serie de actividades; cabe mencionar, que durante este a algunos participantes se les realizó análisis de movimiento de mouse y ojos usando el Software Ogama y eye-tracking. Luego, cada participante debía responder un cuestionario acerca de sus percepciones del juego; en este instrumento se evaluaban aspectos tales como: usabilidad (System Usability Scale), experiencia de usuario (AttrakDiff), estética de la experiencia (VisAWI) y preferencia por el uso.

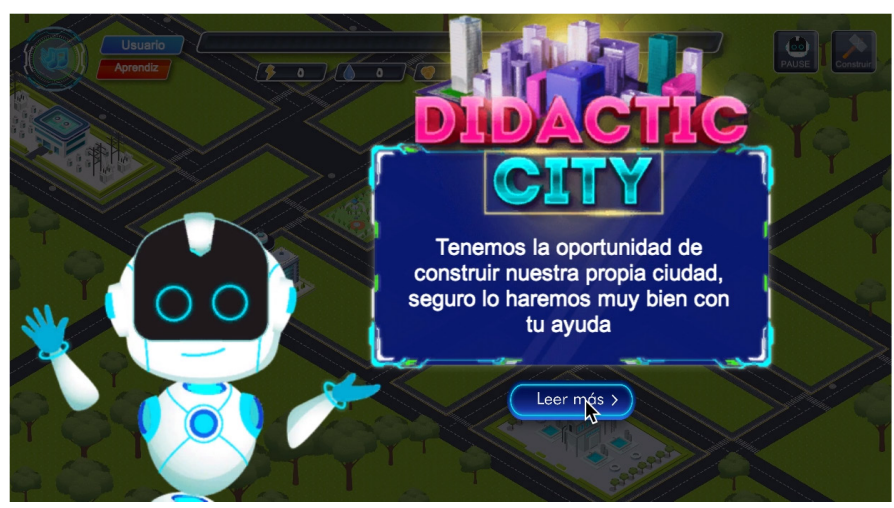

Figura 2. Primer sprint "Didactic City" (Demo)

Los resultados de estas pruebas indicaron que, en general, el demo de "Didactic City" tenía una usabilidad de 42 puntos, estando en un nivel mínimo aceptable. Sin embargo, se hacía necesario la incorporación de un tutorial detallado y claro debido a que los usuarios no captaron con facilidad el funcionamiento del juego. Igualmente, los comentarios de los participantes indicaron la necesidad de especificar los nombres de las edificaciones, aumentar y unificar el tamaño de la letra, entre otros arreglos los cuales se incorporaron en el segundo sprint (Ver Figura 3). Actualmente, se trabaja en el tercer sprint del juego.

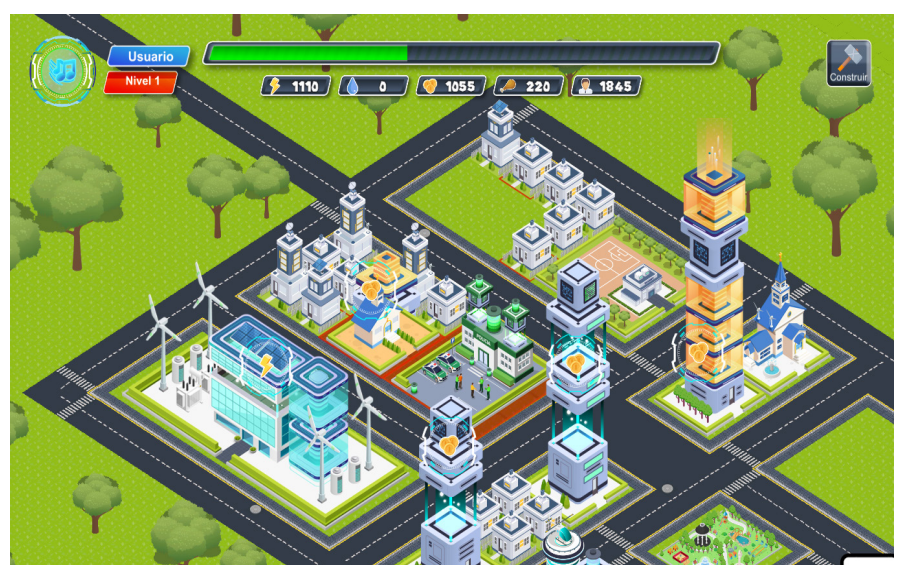

Figura 3. Segundo sprint "Didactic City" 
Para determinar los modelos a implementar se realizó un análisis de viabilidad a los cuatro modelos machine learning supervisados, que de acuerdo a la literatura, son los más usados para la predicción del abandono estudiantil. Para este proceso se utilizó la matriz de decisión que se encuentra en la Tabla 4, en la cual se asigna un peso de 0 a 10 a cada modelo de acuerdo a diversos factores (siendo 10 el mayor valor).

Totalizados los resultados, se determinó que los árboles de decisión y las redes neuronales tenían en conjunto el mejor valor de atributos siendo un factor determinante el uso de lenguajes de programación como Python, el cual dispone de un amplio número de librerías open source que lo hacen ideal para desarrollar modelos machine learning.

Tabla 4. Matriz decisión modelos machine learning

\begin{tabular}{|c|c|c|c|c|}
\hline Factores & $\begin{array}{c}\text { Árbol } \\
\text { decisión }\end{array}$ & $\begin{array}{c}\text { Red neu- } \\
\text { ronal }\end{array}$ & $\begin{array}{c}\text { Regresión } \\
\text { logística }\end{array}$ & $\begin{array}{c}\text { Análisis } \\
\text { discriminante }\end{array}$ \\
\hline Usabilidad & 10 & 10 & 8 & 7 \\
\hline $\begin{array}{c}\text { Requerimiento } \\
\text { datos }\end{array}$ & 9 & 9 & 7 & 7 \\
\hline Adaptabilidad & 9 & 9 & 9 & 9 \\
\hline Precisión & 10 & 10 & 9 & 9 \\
\hline $\begin{array}{c}\text { Lenguaje pro- } \\
\text { gramación }\end{array}$ & 10 & 10 & 10 & 8 \\
\hline Total & 48 & 48 & 43 & 41 \\
\hline
\end{tabular}

Adicionalmente, se determinaron los dominios del modelo, en este caso: abandono del curso, tendencias y comportamiento de los estudiantes en actividades de refuerzo, participación en actividades académicas y análisis de texto en cada actividad. Variables tales como nombre del estudiante, ejercicios, calificaciones, entre otras, se extraen de los LMS y se cargan en el data warehouse, donde se organiza la información en estructuras para el proceso de minería de datos. Cada dominio tiene como input la estructura de datos normalizados y como output las clasificaciones correspondientes en nivel de riesgo o temática, tal como se observa en la Figura 4.

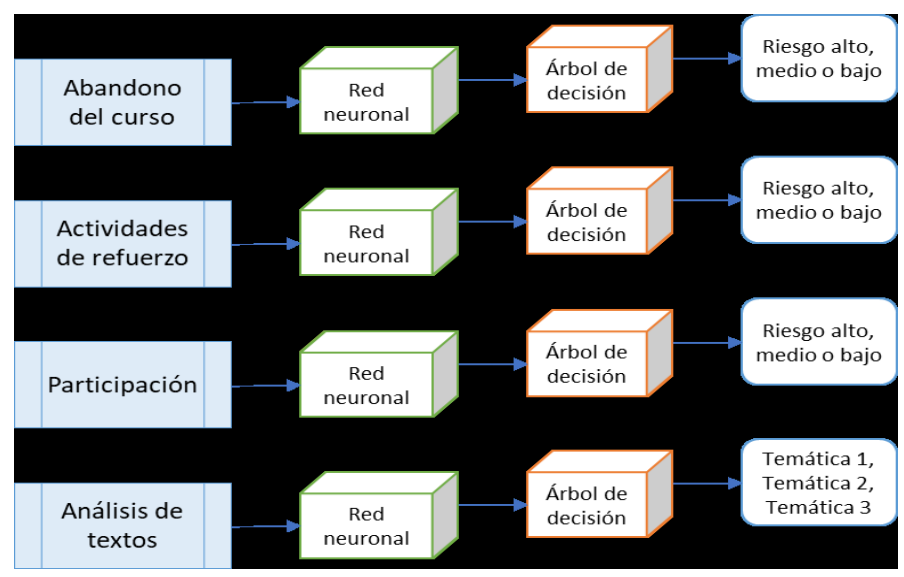

Figura 4. Modelo de Inteligencia Artificial de "Didactic-City"

En este modelo, la red neuronal realiza una combinatoria de cada posible estado de los atributos de entrada con cada posible estado del atributo a predecir, calculando probabilidades que utilizan los datos de entrenamiento para la clasificación y para predecir un resultado. Una parte de los datos de entrenamiento se reservan para evaluar la precisión de la red, por lo que en cada iteración la red se evalúa inmediatamente, arrojando el estatus de los datos procesados. Por su parte, el árbol de decisión, gracias a redes bayesianas, genera internamente divisiones o nodos cada vez que el valor de entrada tiene una correlación significativa con la columna de predicción; en este caso, este algoritmo evalúa las condiciones generadas por la red neuronal con el objetivo de clasificar de forma precisa los datos en los rangos que se requieren. Además, internamente el algoritmo incluye la selección de características para evitar que variables no relevantes utilicen tiempo del procesador.

Actualmente se trabaja en la perfección del modelo, pues teniendo en cuenta que es un modelo supervisado, cuenta con una proyección en porcentaje (\%) de la meta que se quiere lograr en todos los niveles.

En relación al estándar de contactabilidad, el cual actualmente se encuentra en construcción, será automatizado a partir de los resultados en los modelos machine learning y el estado del estudiante en la gamificación activa y pasiva. El contacto unilateral buscará mantener fidelizados a los estudiantes tanto a sus actividades académicas dentro del LMS como a la herramienta "Didactic City". Dicho contacto se hará mediante llamadas telefónicas, mensajes de texto y comunicación electrónica.

El flujo de contacto estará dado por 3 eventos diferentes: (1) Autorización en el que cada usuario, si así lo quiere, puede aceptar los términos, condi- 
ciones y tratamiento de datos personales; (2) Eventos básicos los cuales están relacionados con la interacción del estudiante con el LMS y pueden ser de dos tipos: negativos (por ejemplo, cuando el estudiante tiene tareas vencidas o por vencer) o positivos (por ejemplo, cuando el estudiante está al día con las tareas y participaciones); (3) Eventos alternos los cuales permitirán notificar al usuario sobre su avance en "Didactic City", informándole acerca de las mejoras o reconocimientos obtenidos.

\section{CONCLUSIONES}

La gamificación y la inteligencia artificial son estrategias que poseen múltiples beneficios, por lo que se recomienda seguir implementándolas en entornos virtuales de aprendizaje, pues gracias a estas se puede aumentar la motivación y el engagement, contribuyendo a la disminución del abandono estudiantil y mejorando los procesos de enseñanza y aprendizaje.

Adicionalmente, se recomienda replicar la herramienta gamificada "Didactic City" con temáticas diferentes a las competencias ciudadanas, para que pueda ser aplicada en clases tanto presenciales como virtuales; esto teniendo en cuenta diversas investigaciones en las que se ha demostrado los beneficios que tiene la gamificación en diferentes contextos educativos.

Igualmente, sería interesante continuar con el uso de sistemas similares a los establecidos en la plataforma de contactabilidad, especialmente en la modalidad educativa virtual, pues este sistema no solo permite realizar diferentes predicciones relacionadas con el proceso académico de cada estudiante, sino que facilita tener un mayor acompañamiento por parte de los docentes a los alumnos brindándoles una educación un poco más personalizada.

Por otra parte, para futuras etapas de DIDACTIC se seguirá aplicando monitoreo contante, pues es importante considerar las percepciones de sus usuarios potenciales para obtener mejores resultados. Finalmente, para proyectos similares se recomienda seguir la metodología ágil SCRUM, ya que está permite obtener el mejor resultado posible de proyectos que se desarrollan en contextos complejos y cambiantes que requieren una solución rápida y efectiva.

\section{AGRADECIMIENTOS}

Agradecimiento especial por el apoyo financiero recibido para la realización de este proyecto a la Universidad Industrial de Santander y al Sistema General de Regalías fondos de CTel de la Gobernación de Antioquia administrados a través del Patrimonio Autónomo Fondo Nacional de Financiamiento para la Ciencia, la Tecnología y la Innovación Francisco José de Caldas - MINCIENCIAS-. 


\section{REFERENCIAS}

Acosta-Medina, J. K., Torres-Barreto, M. L., \& Alvarez-Melgarejo, M. (2020). Literature mapping about gamification in the teaching and learning processes. Revista ESPACIOS, 41(11), 26.

Acosta-Medina, J. K., Torres-Barreto, M. L., Álvarez-Melgarejo, M., \& Paba-Medina, M. C. (2020). Gamificación en el ámbito educativo: Un análisis bibliométrico. I+D Revista de Investigaciones, 28-36. https://doi.org/10.33304/revinv.v15n1-2020003

Acosta-Medina, J. K., Torres-Barreto, M. L., Paba-Medina, M. C., \& Alvarez-Melgarejo, M. (2020). Análisis de la gamificación en relación a sus elementos. Retrieved from https://hal.archives-ouvertes.fr/ hal-02548860/document

Alpaydin, E. (2020). Introduction to Machine Learning (Fourth Edi). Cambridege, Massachusetts: The MIT Press.

D’Addario, M. (2019). Inteligencia Artificial: Tratados, Aplicaciones, Usos y Futuro (Amazon Digital Services, ed.).

Dalipi, F., Imran, A. S., \& Kastrati, Z. (2018). MOOC dropout prediction using machine learning techniques: Review and research challenges. IEEE Global Engineering Education Conference, EDUCON, 2018-April, 1007-1014. https://doi.org/10.1109/ EDUCON.2018.8363340

Deterding, S., Dixon, D., Khaled, R., \& Nacke, L. (2011). From game design elements to gamefulness. Proceedings of the 15th International Academic MindTrek Conference on Envisioning Future Media Environments - MindTrek'11, 9.

Dicheva, D., Dichev, C., Agre, G., \& Angelova, G. (2015). Gamification in Education: A Systematic Mapping Study. Educational Technology \& Society, 18(3), $75-88$.

Galipienso, M. I. A., Quevedo, M. A. C., Pardo, O. C., Ruiz, F. E., \& Lozano, M. A. (2015). Inteligencia artificial: modelos, técnicas y áreas de aplicación. Thompson Paraninfo S.A.

Jurado, F., Asbusac, J., \& Sanchez, J. C. (2018). Creación de Videojuegos: Desarrollo de Componentes. BuBok.

Kumar, J. M., \& Herger, M. (2013). Gamification at Work: Designing Engaging Business Software. Interaction Design Fundation.

Lobo-Rueba, M. Á., Paba-Medina, M. C., \& Torres-Barreto, M. L. (2020). Análisis descriptivo de experiencias gamificadas para enseñanza y aprendizaje en educación superior en ingeniería. Revista ESPACIOS, 41(16), 21.
Melo-Solarte, D. S., \& Díaz, P. A. (2018). El Aprendizaje Afectivo y la Gamificación en Escenarios de Educación Virtual. Información Tecnológica, 29(3), 237-248.

Ministerio de Educación Nacional de Colombia. (2006). Estándares Básicos de Competencias en lenguaje, matematicas, ciencias y ciudadanas. Bogotá.

Ministerio de Educación Nacional de Colombia. (2017). Reporte sobre deserción y graduación en educación superior. Bogotá, Colombia.

Ministerio de Educación Nacional de Colombia. (2019). Educación virtual. Bogotá.

Rodrigues, L. F., Oliveira, A., \& Rodrigues, H. (2019). Main gamification concepts: A systematic mapping study. Heliyon, 5(7).

Takeuchi, H., \& Nonaka, I. (1986). The new product development game. Harvard Business Review, 137146.

Viloria, A., Senior Naveda, A., Hernández Palma, H., Niebles Núẽz, W., \& Niebles Núẽz, L. (2020). Using Big Data to Determine Potential Dropouts in Higher Education. Journal of Physics: Conference Series, 1432(1).

Werbach, K., \& Hunter, D. (2012). For the Win: How Game Thinking Can Revolutionize Your Business. Wharton Digital Press. 